NUC-MINN-99/4-T

March 1999

\title{
SIZE OF FIREBALLS CREATED IN HIGH ENERGY HEAVY ION COLLISIONS AS INFERRED FROM COULOMB DISTORTIONS OF PION SPECTRA
}

\author{
Alejandro Ayala ${ }^{1}$ \\ Instituto de Ciencias Nucleares UNAM \\ A.P. 70-543, México D.F. 04510, México \\ Sangyong Jeon ${ }^{2}$ \\ Nuclear Science Division \\ Lawrence Berkeley National Laboratory \\ Berkeley, CA 94720 \\ Joseph Kapusta ${ }^{3}$ \\ School of Physics and Astronomy \\ University of Minnesota \\ Minneapolis, MN 55455
}

\begin{abstract}
We compute the Coulomb effects produced by an expanding, highly charged fireball on the momentum distribution of pions. We compare our results to data on $\mathrm{Au}+\mathrm{Au}$ at $11.6 \mathrm{~A} \mathrm{GeV}$ from E866 at the BNL AGS and to data on $\mathrm{Pb}+\mathrm{Pb}$ at 158 A GeV from NA44 at the CERN SPS. We conclude that the distortion of the spectra at low transverse momentum and mid-rapidity can be explained in both experiments by the effect of the large amount of participating charge in the central rapidity region. By adjusting the fireball expansion velocity to match the average transverse momentum of protons, we find a best fit when the fireball radius is about $10 \mathrm{fm}$, as determined by the moment when the pions undergo their last scattering. This value is common to both the AGS and CERN experiments.
\end{abstract}


PACS numbers: 25.75.-q, 02.70.Ns, 24.10.Nz

1 ayala@xochitl.nuclecu.unam.mx

2 jeon@nta2.lbl.gov

3 kapusta@physics.spa.umn.edu 
The purpose of colliding heavy nuclei at relativistic and ultrarelativistic energies is to produce high density, high temperature matter, either hadronic or quark-gluon plasma. One way of inferring the properties of matter under these extreme conditions is to compare the single particle momentum distributions of secondaries to those obtained in collisions of lighter systems at similar beam energies. An example is provided by the qualitative differences found between the transverse momentum distributions from heavy-ion reactions and those from $\mathrm{p}-\mathrm{p}$ collisions. In the former, the bulk features of the spectrum can successfully be explained by the large amount of secondary scattering, potentially leading to the onset of hydrodynamical behavior [1].

Another feature to account for in the collision of heavy systems is the presence of a large amount of electric charge that will influence the dynamics of secondary charged particles. Due to the long-range nature of the Coulomb field, the spectrum of charged particles can still be distorted even after freeze-out. For central collisions this Coulomb effect can be more significant in the case of strong stopping when the participant charge in the central rapidity region is an important fraction of the initial charge, particularly on the low momentum particles.

In a recent paper [2] two of us explored the influence of the time dependent electric field produced by an expanding, highly charged fireball on the low energy part of the spectrum of charged test particles, taken to be kaons [3]. The analysis was based on the solution to Vlasov's equation assuming that the test particles' dynamics was nonrelativistic. In this paper we extend that analysis, treating the test particle relativistically. We also apply the analysis to the transverse momentum distribution of charged pions and compare the results to recent measurements of $\mathrm{Au}+\mathrm{Au}$ collisions by the E866 collaboration at the $\mathrm{BNL}$ AGS and to recent measurements of $\mathrm{Pb}+\mathrm{Pb}$ collisions by the NA44 collaboration at the CERN SPS. Relativistic calculations for pions and kaons have also been done in somewhat different models by Barz et al. [4].

We briefly recall the basics of the model used in Ref. [2] and how it is modified for relativity. A uniformly charged sphere which has a total charge $Z e$ and whose radius $R$ increases linearly with time $t$ from a value $R_{0}$ at time $t_{0}$ at a constant surface speed $v_{s}$ produces an electric potential

$$
V(r, t)=\left\{\begin{array}{ll}
Z e / 4 \pi r, & r \geq R=v_{s} t \\
Z e\left(3 R^{2}-r^{2}\right) / 8 \pi R^{3}, & r \leq R=v_{s} t
\end{array} .\right.
$$

In the center-of-mass frame of the fireball the charge moves radially outwards, hence there is no preferred direction and consequently the magnetic field produced by this moving charge configuration vanishes. The fireball parameters are related by $R_{0}=v_{s} t_{0}$. If $f^{ \pm}(\mathbf{r}, \mathbf{p}, t)$ represents the $\pm e$ test particle phase space distribution then, when ignoring particle collisions after decoupling, its dynamics is governed by Vlasov's equation.

$$
\left[\frac{\partial}{\partial t}+\frac{\mathbf{p}}{E_{p}} \cdot \nabla_{r} \pm e \mathbf{E}(\mathbf{r}, t) \cdot \nabla_{p}\right] f^{ \pm}(\mathbf{r}, \mathbf{p}, t)=0,
$$


where $E_{p}=\sqrt{p^{2}+m^{2}}, m$ is the meson's mass and

$$
e \mathbf{E}(\mathbf{r}, t)=-e \nabla_{r} V(r, t)= \begin{cases}\left(t_{s} / 4 t^{3}\right) \mathbf{r}, & r \leq R=v_{s} t \\ \left(t_{s} v_{s}^{3} / 4 r^{3}\right) \mathbf{r}, & r \geq R=v_{s} t\end{cases}
$$

is the time-dependent electric field corresponding to the potential $V(r, t)$. Here we have defined the characteristic time $t_{s}$ by

$$
t_{s} \equiv \frac{Z e^{2}}{\pi m v_{s}^{3}}
$$

and we work in units where $c=1$. The set of Eqs. (11)-(4) are relativistically correct. Notice that there is no problem with retardation effects since what is specified in these equations is the electric field and not the charge density. Of course, the relationship between the charge density and the eletric field gets modified due to the finite speed of light, but we are not interested in that relationship here. The only difference between the equations to be solved here and those solved in Ref. [2] involve the energy $E_{p}$ in Eq. (2) which was approximated by $m+p^{2} / 2 m$ in the nonrelativistic case.

The solution to Eq. (2) is found by the method of characteristics. This involves solving the classical equations of motion and using the solutions to evolve the initial distribution, assumed here to be thermal

$$
f^{ \pm}\left(\mathbf{r}, \mathbf{p}, t_{0}\right)=\exp \left\{-\left(E_{p} \pm V\left(r, t_{0}\right)\right) / T\right\}
$$

forward in time. The equations of motion are:

$$
\begin{aligned}
\frac{d \mathbf{r}}{d t} & =\frac{\mathbf{p}}{E_{p}} \\
\frac{d \mathbf{p}}{d t} & = \pm e \mathbf{E}(\mathbf{r}, t) .
\end{aligned}
$$

The pion's asymptotic momentum is calculated numerically by a 6'th order Runge-Kutta method with adaptive step sizes from a set of initial phase-space positions. The final momentum distribution is the result of computing the trajectories for many initial phase space points. The initial radial position was incremented in $\mathrm{N}_{r}=50$ steps with spacing $\Delta r / R_{0}=0.02$. The initial momentum was incremented in $\mathrm{N}_{p}=600$ steps with spacing $\Delta p$ $=1 \mathrm{MeV}$. The cosine of the angle between the initial position and momentum vectors was incremented in $\mathrm{N}_{z}=100$ steps of size $\Delta \cos \theta=0.02$. Hence the total number of trajectories computed was $3 \times 10^{6}$ for each set of initial conditions.

For central $\mathrm{Pb}+\mathrm{Pb}$ collisions at $158 \mathrm{~A} \mathrm{GeV}$ at the SPS, for which NA44 and NA49 have reported a significant amount of stopping [5, 6], the number of protons per unit rapidity in the central region is on the order of 30 . If we consider that the rapidity region spanned by the fireball is between 1 and 5 units, then we take as the effective fireball's charge $Z=120$. 
To estimate the surface velocity $v_{s}$ we note that the average transverse velocities of pions and protons in $\mathrm{Pb}+\mathrm{Pb}$ collisions at the SPS are greater than 0.6 [1]. Therefore, to represent the electric field it is reasonable to take the maximum velocity of charges to be greater than that; we shall use $v_{s}=0.8$ and $v_{s}=1$.

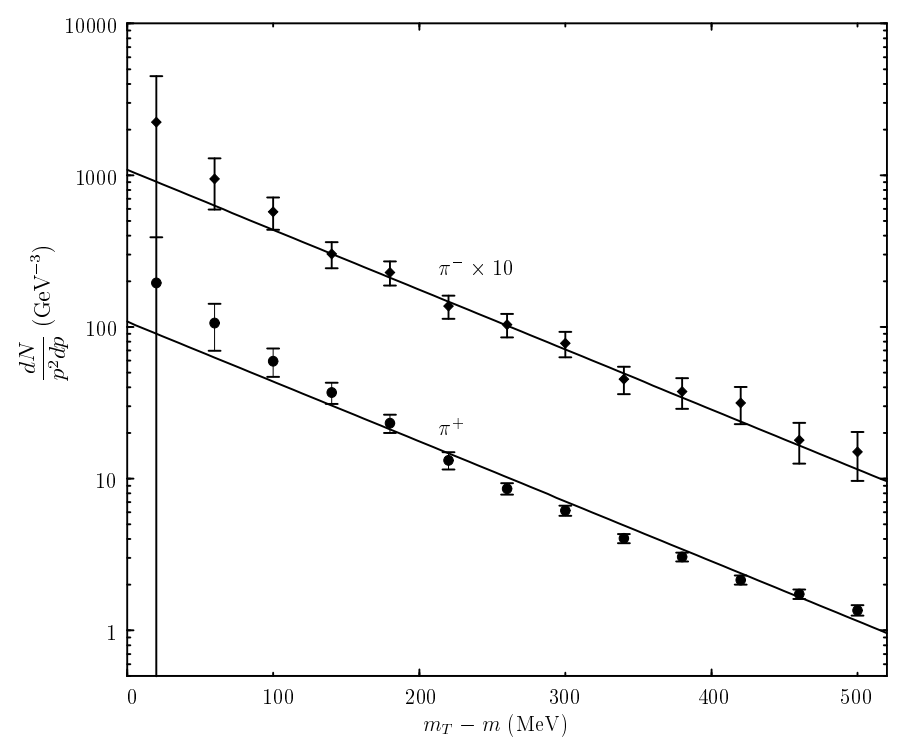

Figure 1: The transverse mass distributions at mid-rapidity for $\pi^{+}$and $\pi^{-}$. The solid lines are exponentials with an inverse slope of $110 \mathrm{MeV}$. The data are from NA44 [14].

The model we are using is spherically symmetric. It has long been known [7] that the momentum distributions at the SPS are somewhat forward-backward peaked, even for central $\mathrm{Pb}+\mathrm{Pb}$ collisions [8]. A spherically symmetric model is, of course, not essential to the basic physics and can be relaxed at the expense of additional computing time. Nevertheless, we can provide the following arguments in favor of its use. First, we will be comparing with the transverse momentum distributions at mid-rapidity where the impact of spherical asymmetry should be less important than near the fragmentation regions. Second, pion interferometry of central $\mathrm{Au}+\mathrm{Au}$ collisions at the AGS [9] and of central $\mathrm{Pb}+\mathrm{Pb}$ collisions at the SPS [10] both yield comparable values for the transverse and longitudinal radii at the time of pion freezeout or strong decoupling. These are about twice the radius of a cold gold or lead nucleus. Third, we shall show later that the transverse surface of the fireball must expand outwards with a speed more than $90 \%$ that of light in order to reproduce the average proton transverse momentum. Since the longitudinal surface of the fireball cannot travel faster than the speed of light, this means that in velocity space the fireball is nearly symmetric. These phenomena, although not yet measured at that time, 
were already known to Landau [11]. His model envisioned the two nuclei stopping each other within one Lorentz contracted nuclear diameter, then undergoing a one dimensional expansion along the beam axis. After a time of order (nuclear radius)/c the longitudinal size of the fireball had become comparable to the transverse size and, furthermore, the rarefaction wave would have caused transverse expansion to set in. As a rough approximation one therefore could imagine the original one dimensional expansion to become three dimensional after that time. This model was later developed by others, including Cooper, Frye and Schonberg [12]. The essential insight from Landau's model is not the degree of stopping but rather the point that significant transverse expansion sets in after the longitudinal and transverse radii become comparable in magnitude. Thereafter, from the point of view of a distant observer, the expansion is not as asymmetric as one might originally think.

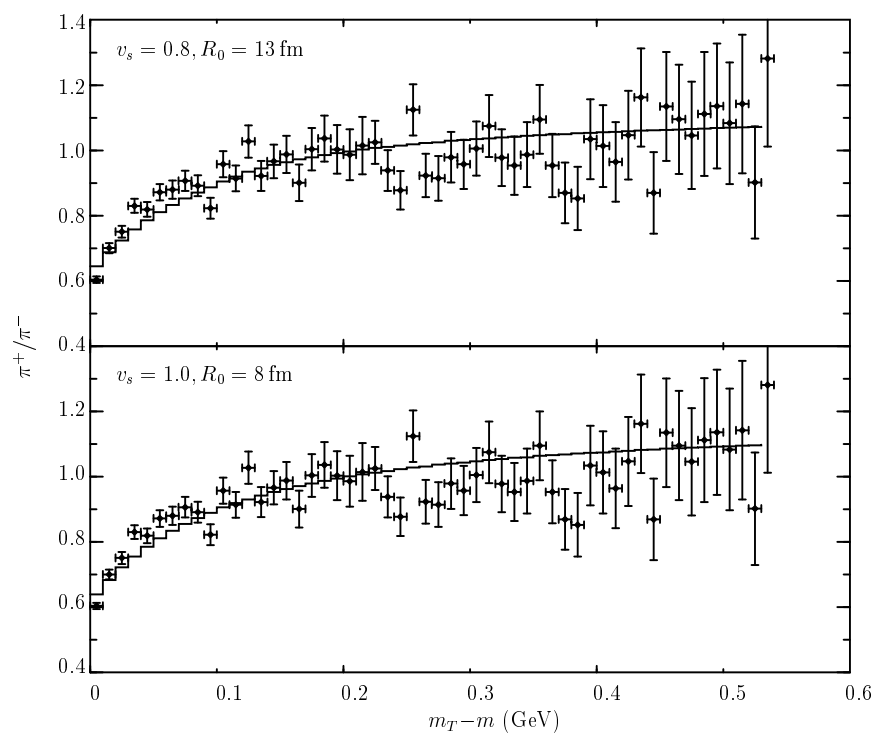

Figure 2: The ratio $\pi^{+} / \pi^{-}$vs. $m_{T}-m$ at the SPS. The data is from [5]. Top panel: The curve corresponds to the Coulomb calculation described in the text with surface velocity 0.8 and initial radius $13 \mathrm{fm}$. Bottom panel: Same as top panel but with the parameters 1 and $8 \mathrm{fm}$, respectively.

The collaboration NA44 has reported a suppression of the $\pi^{+} / \pi^{-}$ratio as $p_{T} \rightarrow 0$ in $\mathrm{Pb}+\mathrm{Pb}$ collisions, presumably due to the Coulomb field of the fireball. Only a very small, or no, suppression is observed in $\mathrm{S}+\mathrm{Pb}$ collisions. To model the primordial distribution we use an exponential parametrization of $d N / p^{2} d p$. Fig. 1 shows this representation [13] in comparison to the transverse mass distributions of positive and negative pions [14] with 
a value of $T_{\text {eff }}=110 \mathrm{MeV}$. This common fit (apart from the absolute normalization) is then used to model the initial pion distribution, and the Coulomb computation we do is a perturbative correction to it. In essence we sprinkle the pions around at time $t_{0}$ according to a relativistic Boltzmann distribution with an effective temperature of $110 \mathrm{MeV}$ and solve the Vlasov equation for subsequent time. This assumes that all resonances that can decay into charged pions have already done so, hence their effects are contained in this parametrization. Even most of the relatively long-lived omega-mesons should have decayed by the this late freeezeout time and relatively low temperature.

The procedure we follow is to fix $v_{s}$ at either 0.8 or 1 and then vary $R_{0}$ to get the best fit to the ratio $\pi^{-} / \pi^{+}$as a function of $m_{T}-m$ in the range from 0 to $500 \mathrm{MeV}$. The data's bin size is $10 \mathrm{MeV}$. The data are shown in Fig. 2 and compared to the results of calculations with $v_{s}=0.8, R_{0}=13 \mathrm{fm}$ and $v_{s}=1, R_{0}=8 \mathrm{fm}$. There is no visual difference between the calculations with the two parameter sets. The reason the final Coulomb corrected ratio is nearly identical for these two sets of parameters is easily understood. According to the study in Ref. [2], the longer the test charge stays in the electric field of the fireball the more it is modified from its initial form. Thus, for the more slowly expanding fireball $\left(v_{s}=0.8\right)$ the radius must be larger $(13 \mathrm{vs} .8 \mathrm{fm})$ in order to reduce the magnitude of the electric field so as to represent the data.

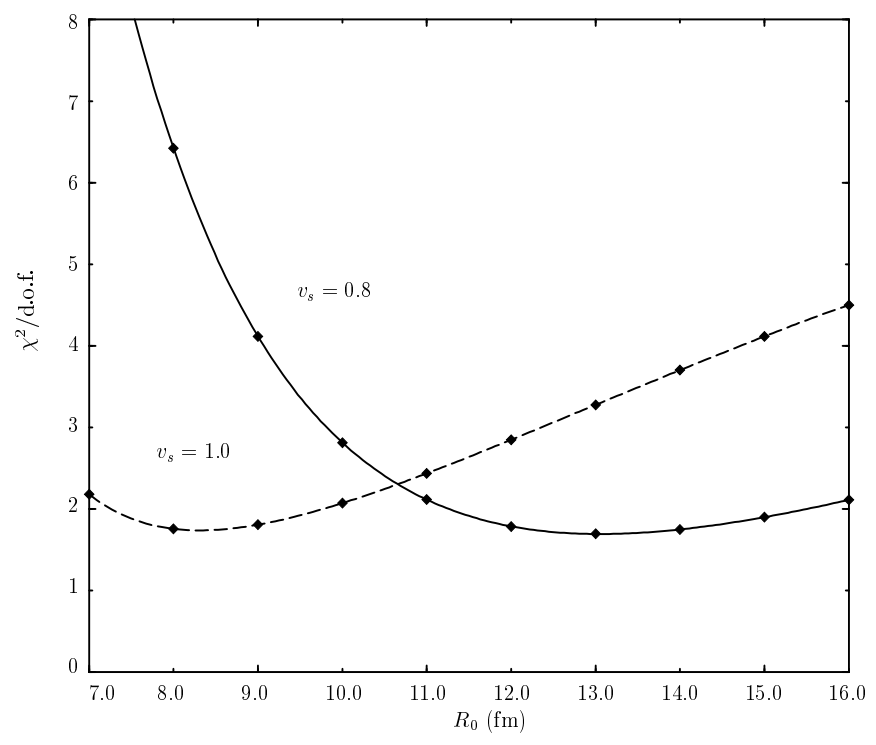

Figure 3: Chi-squared vs. radius for fixed values of the surface velocity for pions for central $\mathrm{Pb}+\mathrm{Pb}$ collisions at the SPS. The curves represent polynomials which pass through the values of $R_{0}$ for which computations were done. 
In Fig. 3 we plot the chi-squared for the $\pi^{+} / \pi^{-}$ratio as a function of radius $R_{0}$ for the surface velocity $v_{s}$ fixed at 0.8 and 1 . The minimum is achieved at about 13 and $8.3 \mathrm{fm}$, respectively. The difference in the chi-squared for the two values of $v_{s}$ is not significant enough to make a judgement on which gives the better fireball physics. The reason for the close coupling of the two parameters, $v_{s}$ and $R_{0}$, is explained by recognizing that it is the ratio of the characteristic expansion time $t_{0}$ to the characteristic Coulomb time $t_{s}$ which is the relevant quantity. Since $t_{0} / t_{s} \propto v_{s}^{2} R_{0}$ we would expect comparable fits to the data will be obtained when $R_{0}$ varies inversely with the square of $v_{s}$; this is borne out by our explicit numerical calculations. We remark that a different parametrization of the primordial pion distribution, such as a two temperature fit, might well lead to an even better representation of the data.

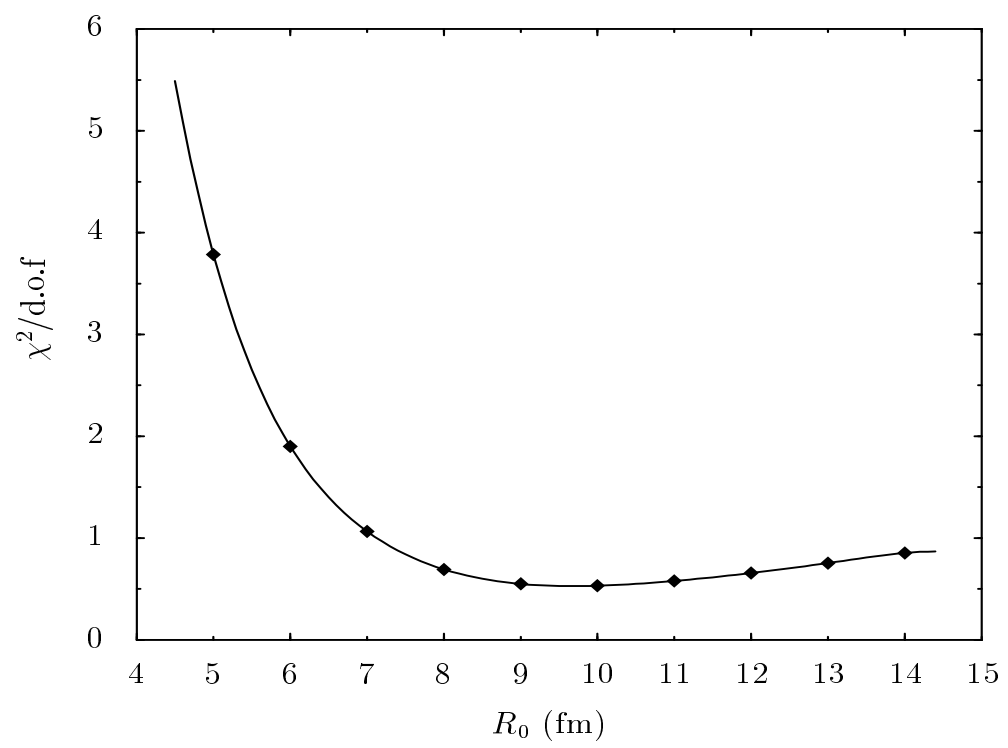

Figure 4: Chi-squared vs. radius for a surface velocity of 0.914 for pions for central $\mathrm{Au}+\mathrm{Au}$ collisions at the AGS. The curves represent polynomials which pass through the values of $R_{0}$ for which computations were done.

The immediate question now is how to determine the appropriate value of $v_{s}$. We shall do this by matching the average transverse momentum of the protons as inferred from our assumption of a uniformly expanding sphere with the measured one. The model gives

$$
\left\langle p_{T}\right\rangle=\frac{\int d^{3} r \rho(r, t) \sin \theta m_{P} v / \sqrt{1-v^{2}}}{\int d^{3} r \rho(r, t)},
$$


where $\rho(r, t)=\rho_{0}\left(t_{0} / t\right)^{3} \Theta\left(v_{s} t-r\right)$ and $v=r / t$. The result is:

$$
\left\langle p_{T}\right\rangle=\pi \frac{2-\left(2+v_{s}^{2}\right) \sqrt{1-v_{s}^{2}}}{4 v_{s}^{3}} m_{P} .
$$

In the limits $v_{s} \rightarrow 0$ and $v_{s}=1$ one gets $\left\langle p_{T}\right\rangle=3 \pi m_{P} v_{s} / 16$ and $\left\langle p_{T}\right\rangle=\pi m_{P} / 2$, respectively. Note that $v_{s}$ should not be interpreted as a hydrodynamic flow velocity. Rather, it embodies the combined effects of hydrodynamic flow and thermal motion of the net charge carriers, mainly protons. Both NA44 [14] and NA49 [15] have reported the transverse mass distribution in central $\mathrm{Pb}+\mathrm{Pb}$ collisions at midrapidity to be $d N / p_{T} d p_{T} \propto$ $\exp \left(-m_{T} / T_{P}\right)$ with $T_{P}=290 \mathrm{MeV}$. This corresponds to an average transverse momentum of $825 \mathrm{MeV}$. The value $v_{s}=0.916$ gives the best matching of the model to the proton spectra. This finally pins down the freezeout radius for pions to be $10 \mathrm{fm}$.

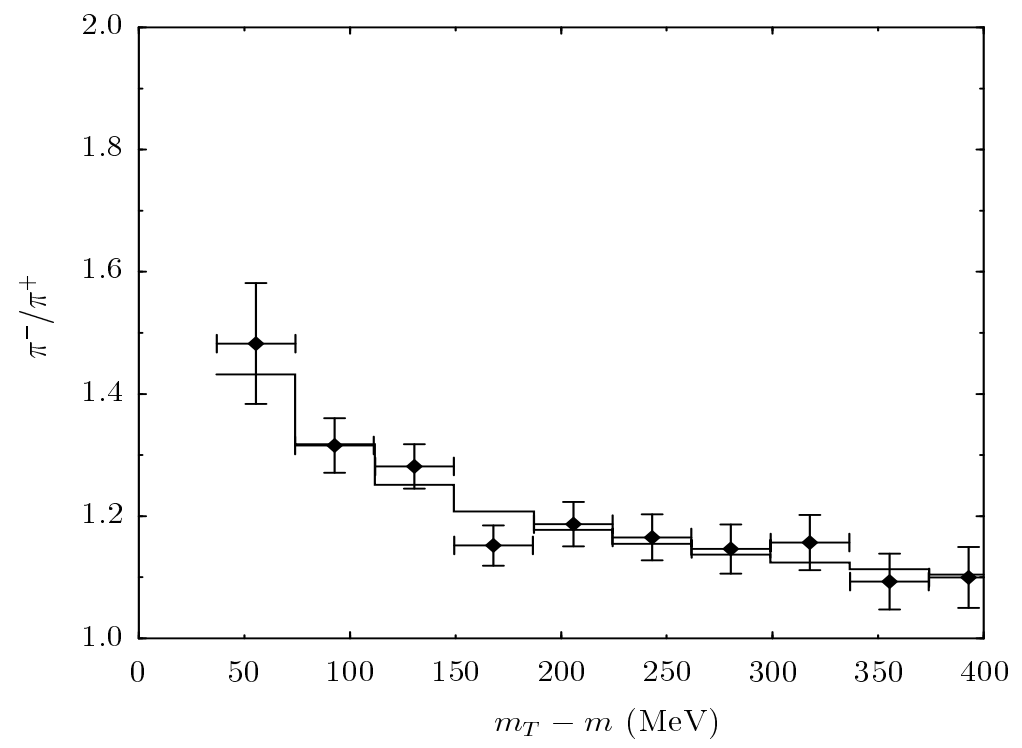

Figure 5: The ratio $\pi^{-} / \pi^{+}$vs. $m_{T}-m$ at the AGS. The data is from [16]. The fireball radius at pion freezeout is $10 \mathrm{fm}$.

We now repeat the above analysis for the BNL experiment E866 [16]. This experiment measured protons and positive and negative pions for $\mathrm{Au}+\mathrm{Au}$ collisions at $11.6 \mathrm{~A} \mathrm{GeV}$. We focus on the most central events, those with the highest multiplicity and transverse energy. The average proton transverse momentum is $820 \mathrm{MeV}$ yielding the surface speed $v_{s}=0.914$. The number of participating protons maybe be estimated from the data to be $Z=116$. The slopes of the $\pi^{+}$and $\pi^{-}$are the same to within several $\mathrm{MeV}$; their average is $111 \mathrm{MeV}$. All of these quantities are remarkably similar to those in central $\mathrm{Pb}$ collisions at the much higher energy of the SPS. 
The experiment reported the ratio $\pi^{-} / \pi^{+}$for a central window of rapidity. We calculated the ratio with two parameters: the radius at pion freezeout $R_{0}$ and the absolute normalization of the individual spectra. The chi-square per degree of freedom is shown in Fig. 4 as a function of $R_{0}$. It has a minimum of 0.53 near $10 \mathrm{fm}$, the same as $\mathrm{Pb}$ collisions at the much higher SPS energy! The lower value of chi-square in this case as compared to the SPS data may be due to the better approximation of spherical symmetry. The calculated ratio is compared to the data in Fig. 5 and its appearance is quite satisfactory.

In conclusion, we have demonstrated that the suppression of the ratio $\pi^{+} / \pi^{-}$in central $\mathrm{Pb}+\mathrm{Pb}$ collisions at the SPS as observed by NA44 and in central $\mathrm{Au}+\mathrm{Au}$ collisions at the AGS as observed by E866 can be quantitatively understood as a Coulomb effect. The large electric fields generated by the expanding fireballs seem to distort the pion ratios at small transverse momenta and midrapidity in accordance with these experiments. In principle these ratios should provide a very good measure of the size of the fireball at the time pions cease collisions and begin to free-stream. From experiments [1] one may estimate that about 2500 baryons and mesons are produced with rapidities within 2 units of mid-rapidity in the central $\mathrm{Pb}+\mathrm{Pb}$ collisions. Associating these with a fireball of radius $10 \mathrm{fm}$ means an average density in the fireball reference frame of $0.60 / \mathrm{fm}^{3}$; this is 4 times nuclear density! Taking into account relativistic length contraction the local density is smaller by a factor

$$
\frac{3}{R_{0}^{3}} \int_{0}^{R_{0}} d r r^{2} \gamma(r)=\frac{3}{2 v_{s}^{2}}\left[\sin ^{-1}\left(v_{s}\right)-v_{s} \sqrt{1-v_{s}^{2}}\right] .
$$

For $v_{s}=0.916$ this implies a local density of $0.42 / \mathrm{fm}^{3}$, still quite considerable. This implies that at the moment that free-streaming of mesons begins the hadronic density is still interestingly high.

To make further progress it is clear that more accurate data are called for but, more importantly, one should also compute the influence of the Coulomb force within an event simulator which takes into account a more accurate description of the space-time evolution of the collision.

\section{Acknowledgements}

The authors wish to thank $\mathrm{Nu} \mathrm{Xu}$, P. Jacobs and J. Pisut for insightful comments and suggestions. This work was supported by the U.S. National Science Foundation under grant NSF PHY94-21309, by the U.S. Department of Energy under grants DE-FG0287ER40328, DE-AC03-76SF00098 and DE-FG03-93ER40792, and by the CONACyTMéxico under grant I27212-E.

\section{References}


[1] Good overviews are provided by the proceedings of the Quark Matter conferences, the most recent in print being Quark Matter '97, Nucl. Phys. A638, (1998).

[2] A. Ayala and J. Kapusta, Phys. Rev. C 56, 407 (1997).

[3] Similar studies were carried out independently by V. Koch, Nucl. Phys. A590, 531c (1995).

[4] H. W. Barz, J. P. Bondorf, J. J. Gaardhøje, and H. Heiselberg, Phys. Rev. C 56, 1553 (1997); ibid. 57, 2536 (1998).

[5] NA44 Collaboration, H. Bøggild et al., Phys. Lett. B372, 339 (1996).

[6] NA49 Collaboration, T. Wienold et al., Nucl. Phys. A610, 76c (1996).

[7] KLM Collaboration, H. von Gersdorff et al., Phys. Rev. C 39, 1385 (1989).

[8] NA49 Collaboration, P. G. Jones et al., Nucl. Phys. A610, 188c (1996).

[9] E877 Collaboration, J. Barrette et al., Nucl. Phys. A610, 227c (1996).

[10] U. Wiedemann, B. Tomasik and U. Heinz, Nucl. Phys. A638, 475c (1998).

[11] L. D. Landau, Izv. Akad. Nauk SSSR (Physics Series) 17, 51 (1953); S. Z. Belenkij and L. D. Landau, Nuovo Cim. Suppl. 3, 15 (1956).

[12] F. Cooper and G. Frye, Phys. Rev. D 10, 186 (1974); F. Cooper, G. Frye and E. Schonberg, ibid. 11, 192 (1975).

[13] It is also possible to represent transverse momentum distributions as arising from a combination of temperature and hydrodynamic flow, as illustrated more recently by S. Esumi, S. Chapman, H. van Hecke and N. Xu, Phys. Rev. C 55, R2163 (1997). What matters most in the Coulomb analysis is the momentum distribution of mesons at the moment of last collision. We have investigated what happens when a combination of flow velocity and temperature is used to represent the kaons' momentum distributions and it makes very little difference. In fact, the only difference arises from the spatial distribution of mesons at the moment of last collision. Such fine detail is outside the scope of this study.

[14] NA44 Collaboration, N. Xu et al., Nucl. Phys. A610, 175c (1996). There is a slight difference in the numerical value of $T_{\text {eff }}$ depending on whether it is $d^{3} N / d^{3} p$ or $E d^{3} N / d^{3} p$ which is fit with an exponential.

[15] NA49 Collaboration, G. Roland et al., Nucl Phys. A638, 91c (1998).

[16] E866 Collaboration, L. Ahle et al., Phys. Rev. C 57, R466 (1998). 